\title{
Condiciones para el acceso universal a la salud en América Latina: derechos sociales, protección social y restricciones financieras y políticas
}

\author{
Conditions for universal access to health in Latin America: \\ social rights, social protection and financial and political constraints
}

Abstract After a brief review of the concept of health equity and its social and sectoral determinants, some macroeconomic aspects of health expenditure in Latin America are considered. Given the significant contemporary tensions with re gard to social rights and the definition of health benefits, three emblematic experiences are analyzed in very different health systems, namely those of Chile, Colombia and M exico. They cover different aspects, such as the guarantee of health benefits, the reduction of forms of implicit rationing and/or barriers to admission, and also aspects related to the quality of services.

Key words Health equity, Legal aspects, Right to health, Social protection, Latin America
Resumen Tras una sucinta problematización de la equidad en salud, sus determinantes sociales y sectoriales, se analizan aspectos macroeconómicos del comportamiento reciente del gasto en salud en la región. Dadas las importantes tensiones contemporáneas respecto de los derechos y la definición de prestaciones de salud, se tratan tres experienciasemblemáticas, desistemas desalud muy diversos: Chile, Colombia y M éxico. Ellas abarcan distintos aspectos: la garantía de las prestaciones, la reducción de formas de racionamiento implícitas y/o de barreras de entrada, o bien aspectos de calidad.

Palabras clave Equidad en salud, Judicialización, Derecho a la Salud, Protección social, America Latina 


\section{Introducción}

No toda diferencia sanitaria entre grupos expresa una desigualdad en salud. No lo es la variación natural, biológica; tampoco la resultante de un comportamiento perjudicial que ha sido elegido libremente. Son desigualdades aquellas diferencias entre grupos de la población que son injustas, evitables einnecesarias ${ }^{1}$.

Los grados de equidad van a estar determinados originalmente por los modelos de aseguramiento y definanciamiento dela sal ud. Laigualdad de acceso a los servicios para quienes posean iguales necesidades, independientemente de sus ingresos y de su capacidad de pago, está íntimamente relacionada con la vigencia de los principios de solidaridad, universalidad y equidad. La falta de transparencia y las imperfecciones de mercado en razón de la competencia imperfecta, límites de la soberanía del consumidor, asimetrías de información, diversos mecanismos de exclusión, atomización y las dificultades de asociación que enfrentan los consumidores requieren regular los mercados de aseguramiento y prestaciones. Por su parte, las transiciones de mográficas ponen sobrela mesa tapete cómo atender las necesidades de los diferentes grupos de edad en materia de protección social y cuidado.

La distribución del ingreso, los efectos distributivos de la política económica, la generación de empleo de calidad influyen de manera determinante en la calidad de las condiciones de vida $y$, con ello, en el disfrute de la salud, que excede largamentelas políticas sectoriales. El crecimiento económico y desempeño económico y su volatilidad, la el evada desigualdad de la distribución del ingreso, el acceso a infraestructura bási$\mathrm{ca}$, o los shocks asociados con desastres naturales son fundamentales. Para actuar sobre los de terminantes y propiciar sinergias entre diversas políticas, es crucial incorporar estrategias intersectoriales en un entorno macroeconómico establey propicio al crecimiento y a una mejor distribución de los frutos del desarrollo. A su vez, las acciones intersectoriales pueden retroalimentar favorablemente la dinámica del sector planteando nuevos retos. Por ejemplo, cuando las transferencias monetarias a hogares pobres se supeditan a controles de salud ${ }^{2}$, como lo ha evidenciado el programa O portunidades en M éxico ${ }^{3}$.

De forma muy diversa en los países, la segmentación de prestaciones de los sistemas de salud refleja patrones discriminatorios que dificultan intervenciones eficaces en función de los costos y del uso eficiente de recursos. En aras de la equidad es necesario trabajar simultáneamente en las áreas de gestión, financiamiento, aseguramiento y prestación de servicios ${ }^{4}$. La profundidad deese aserto se evidencia con el hecho de que incluso cuando mejoran indicadores como la mortalidad infantil o la esperanza de vida, algunas brechas de desigualdad pueden persistir, e incluso ampliarse. Por ejemplo, en Chile, las brechas de mortalidad infantil considerando grupos educacionales extremos 5 . 0 en Colombia, donde habiendo mejorado la atención prenatal y médica del parto en los últimos años y disminuido las disparidades subnacionales, persisten inequidades muy importantes en cuanto a las complicaciones del parto y posparto ${ }^{6}$.

El cariz de las reformas de salud de la región emprendidas desde la década de los ochenta evidencia muchos matices. La reforma más radical en términos de socavar los principios de solidaridad y de universalidad la introdujo la dictadura militar en Chile, sistema cuyos grados de solidaridad han sido incrementados desde la última década. En su antípoda, desde la transición a la democracia el financiamiento en salud y no contributivo tuvo un claro ímpetu universal en Brasil; el horizonte universal fue importante en $\mathrm{Co}$ lombia, donde desde un aseguramiento bajísimo (en torno al 20\%) se dio un enorme salto con una ley que introduce competencia tanto en el nivel de aseguramiento como de la prestación y contempla dispositivos para la solidaridad. La complejidad del financiamiento y de las combinaciones público privadas alerta a no originar "tierras de nadie" en lo preventivo, como lo demuestra la experiencia colombiana.

Por su parte, los países más rezagados han enfatizado el mayor acceso a servicios de atención primaria. En los sistemas contributivos de baja cobertura, un desafío es vencer resistenciase inercias enquistadas dentro de los propios aparatos para adentrarse en reformas polémicas, como la ruptura de los techos de las cotizaciones en Nicaragua y más aún en Honduras, donde están por debajo del salario mínimo. Las encuestas de hogares muestran que hay ocupados en el sector informal u hogares receptores de remesas con capacidad para cotizar, de existir un régimen de aseguramiento subsidiado?.

Algunas reformas han promovido la competencia en el aseguramiento y la prestación, en diferentes combinaciones público-privadas. Es el caso de Colombia y también de Argentina, con la denominada desregulación de las O bras Sociales. Otro ámbito muy importante tiene quever con la interacción del sector con las reformas de descen- 
tralización. En Brasil, por ejemplo, la reforma universal no tuvo un carácter contributivo y los municipios son muy importantes en el financiamiento de prestación de servicios. En M éxico, el Seguro Popular en Salud también está relacionado con la descentralización desdeel punto devista del financiamiento y la prestación de servicios.

Tal como lo ha formulado la Comisión Económica paraAmérica Latina y el Caribe (CEPAL), parafortalecer y expandir el aseguramiento delos riesgos de salud y alcanzar niveles más elevados de solidaridad es central la integración del financiamiento de los sistemas. Se debe evitar la selección de riesgos y progresar hacia coberturas equitativas aseguradas, que combinen financiamiento contributivo y no contributivo. En segundo lugar, es necesaria una mayor eficiencia mediante contención de costos, mecanismos de compra apropiados y regulación. Por otra parte, se requieren políticas desalud pública, entrelas quese destacan la expansión de la atención primaria y una adecuada coordinación de servicios descentralizados que incluya la compensación de brechas entre regiones. Si bien los países de la región presentan diversas formas y grados de interrelación entre el financiamiento contributivo y no contributivo en salud, una mayor integración entrela seguridad social y el sistema público permite mayores sinergias. Asimismo, la articulación entrela seguridad social y los sistemas públicos puede tener un efecto positivo en la eficiencia si la integración de los subsistemas trae consigo un mejor aprovechamiento de la capacidad instalada y facilita la racionalización mediante mejoras en la gestión y administración delos subsistemas ${ }^{8}$.

\section{Tendencias del gasto en salud}

Los mercados laborales de la región no han podido ser una puerta de entrada universal y dinámica a la protección social. De allí que ésta no pueda-ni deba - centrarseexclusivamenteen sistemas contributivos, y que la solidaridad requie ra mecanismos adicionales $5^{8,9}$.

El gasto en salud, evidentemente, no escapa a las restricciones fiscales y contributivas. $\mathrm{Ha}$ aumentado de manera sostenida pero moderada. En algunos casos incluso se incrementó de manera importante desde 1990. Las siguientes cifras ilustran tendencias nacionales, no comparables entre países, dado que se agregan diferentes niveles de gobierno (Gráfico 1).
Dentro de una tendencia general al alza del gasto desde los noventa, la gran heterogeneidad es evidente. Los incrementosfueron excepcionales, y en unos pocos casos se estanca. En muchos países es persistentemente bajo. En algunos casos, de 2007 a 2008 o en 2009 se evidencia un esfuerzo especial.

En cuanto a su prioridad macroeconómica, considerando la evolución del producto interno bruto (Gráfico 2), el esfuerzo por incrementar el gasto ha sido insuficiente. En varios países la tendencia es errática, y en pocos el incremento es sostenido. H asta el 2005 (Gráfico 3) su prioridad fiscal fue menor que la de otros sectores sociales tales como educación, asistencia social y, especialmente, seguridad social. M edido como porcentaje del gasto público total de los países de la región, se incrementó moderadamente en una década y media, pero su peso sufrió ciertas disminuciones. A diferencia de los cálculos previos que abarcan hasta el 2008, la información actualmente disponibleimpide actualizar esta comparación intersectorial.

Visto a la luz dela volatilidad del crecimiento económico, el gasto en salud ha sido muy procíclico. Cuando acontecieron crisis económicas en los períodos 1994-1998 y 2001-2003, fue muy vulnerable. Los ajustes acontecen con cierto rezago: tienden a hacerse al año siguiente. El gasto fuemenos pro-cíclico en 2006 y en lo quese proyecta del 2009 (Gráfico 4).

El gasto público en salud es levemente progresivo. Los sectores de menores recursos se benefician más de la atención primaria y ambulatoria, indicando la mayor cobertura y el acceso muchas veces gratuito a este tipo de servicios. La atención hospitalaria es más regresiva ${ }^{10}$.

Las personas desprovistas de aseguramiento y sin mayor acceso a servicios de salud tampoco tienen capacidad de pago para cubrir al menos al gunas de sus necesidades, como parece indicarlo el al to gasto reprimido debolsillo en salud en la región ${ }^{11}$. Los hogares efectúan gastos de bolsillo, cuya diversa magnitud en relación con el PIB se muestra en el Gráfico $5^{12}$. Esta magnitud requiere interpretarse y puede ser muy disímil: subyacen factores que van desde la baja cobertura del ase guramiento y delos sistemas públicos de salud en países como H onduras, Nicaragua y Guatemala, hasta el incentivo en Brasil a que sectores de altos ingresos efectúen altos gastos de bolsillo en salud a prestadores privados, que les son "reembolsados" posteriormente al deducirlos de sus contribuciones al impuesto sobre la renta. 


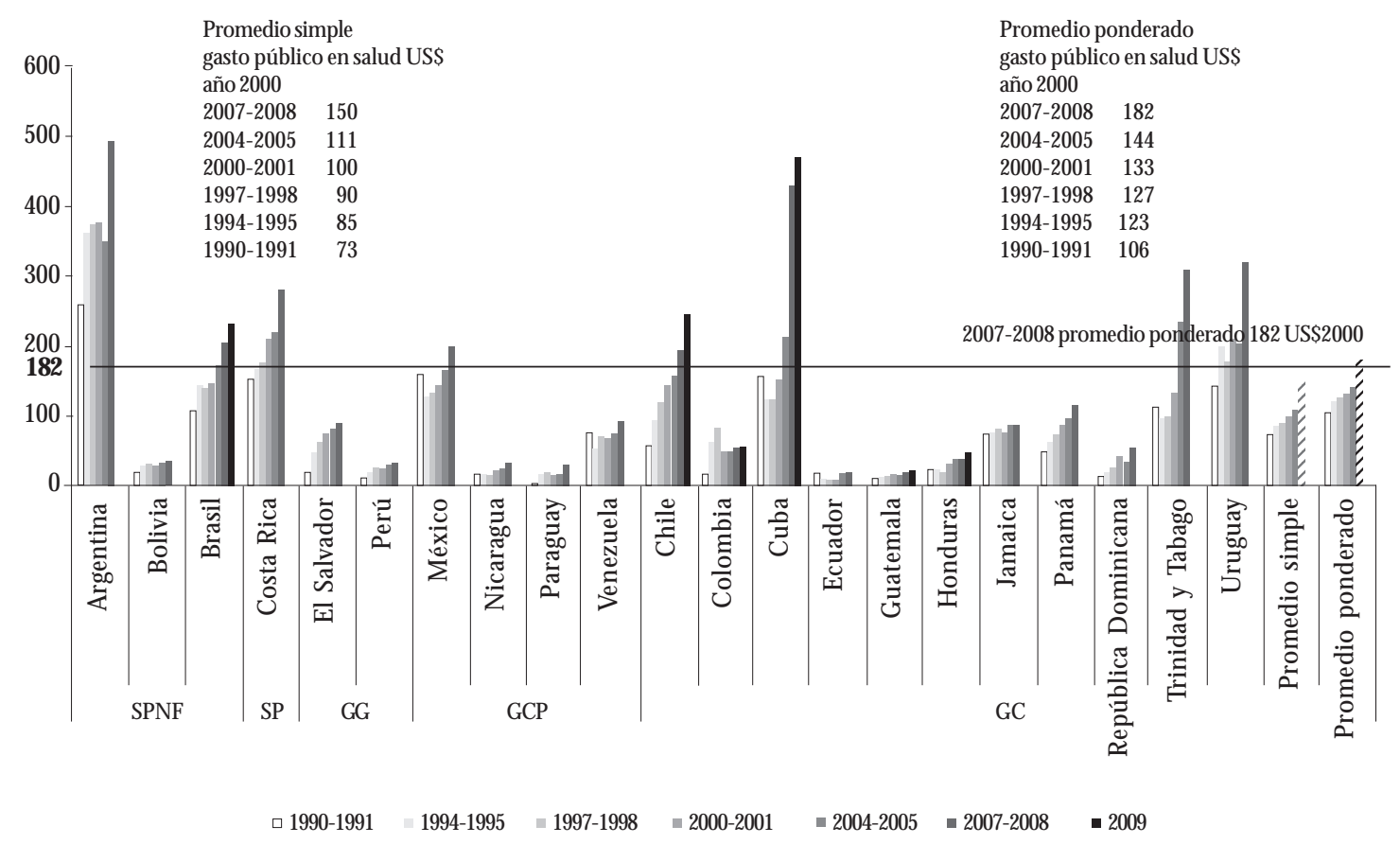

Gráfico 1. América Latina y el Caribe (21 países): gasto público social en salud por habitante (en dólares de 2000).

Según la cobertura institucional de las series de gasto social utilizadas, los países se clasifican así:

Sector público total $=$ SPNF + EPF: Costa Rica

Sector público no financiero $=\mathrm{GG}+$ EPNF: Argentina, Bolivia y Brasil

Gobierno general $=\mathrm{GC}+\mathrm{GL}$ : Perú y El Salvador

Gobierno central = GCP + EA:Colombia, Chile, Cuba, Ecuador, Guatemala, Honduras, Jamaica, Panamá, República Dominicana, Trinidad y Tabago yUruguay.

Gobierno central presupuestario: M éxico, Nicaragua, Paraguay y Venezuela

Donde: EA: entidades estatales autónomas; GL: gobiernos locales; EPNF: empresas públicas no financieras; EPF: empresas públicas de carácter

financiero. M ás detalles en CEPAL, Panorama social 2010, recuadro II.2 (en prensa).

Fuente: CEPAL, con base de datos de gasto social ${ }^{12}$.

Derechossociales, sus garantías y su judicialidad

La ciudadanía política y civil, por una parte, y la social, por otra, tienen supuestos, lógicas y formas organizativas distintas. Las dos primeras coinciden en grandes rasgos con los derechosa la representación y a la participación y nacen en la época moderna vinculadas con un proceso de autonomización de las esferas social y política respecto de la económica.

Sus reglas jurídicas son instrumentales, definen modalidades de acción y de comportamiento, pero no atribuyen medios a cada uno para lograr sus propios fines. Pero la lógica de los dere- chossociales es interdependiente de la economía. Al reinstalar el carácter comunitario de los recursos que constituyen la riqueza nacional, hacen quelos fines delajusticia devengan decisivos para definir los instrumentos y procedimientos pertinentes. La objetivación y formalización de la ciudadanía social, por tanto, implica la temática de quién decide, del cómo se decidey del por quése decide ${ }^{13}$.

Los derechos son indivisibles, tanto en un sentido ético como práctico, en la medida que se refuerzan mutuamente. Los civiles y políticos son indispensables para controlar el cumplimiento de las obligaciones que emanan de los derechos sociales. 


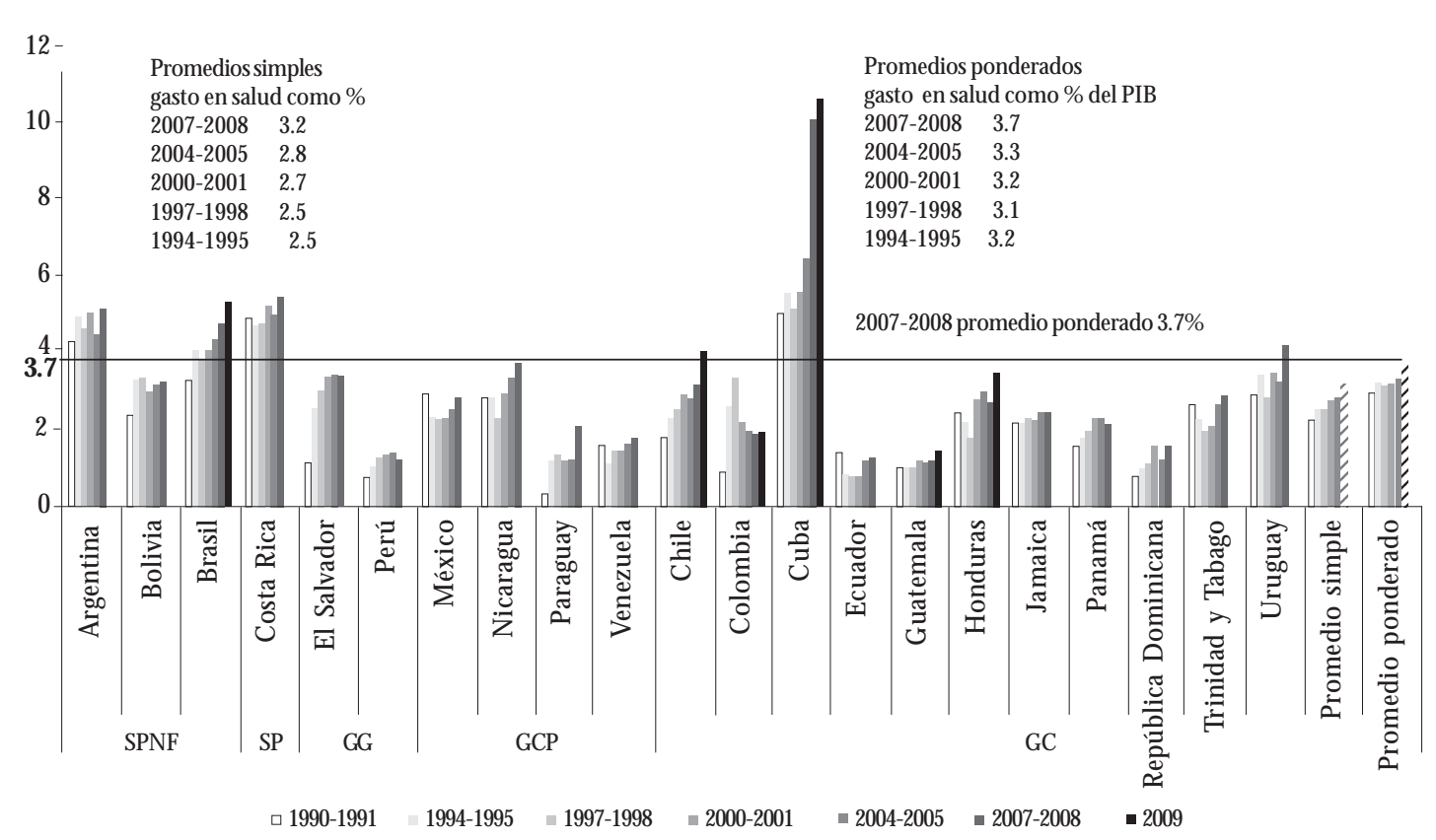

Gráfico 2. América Latina y el Caribe (21 países): gasto público social en salud como \% del PIB.

Fuente: CEPAL, con base de datos de gasto social ${ }^{12}$.

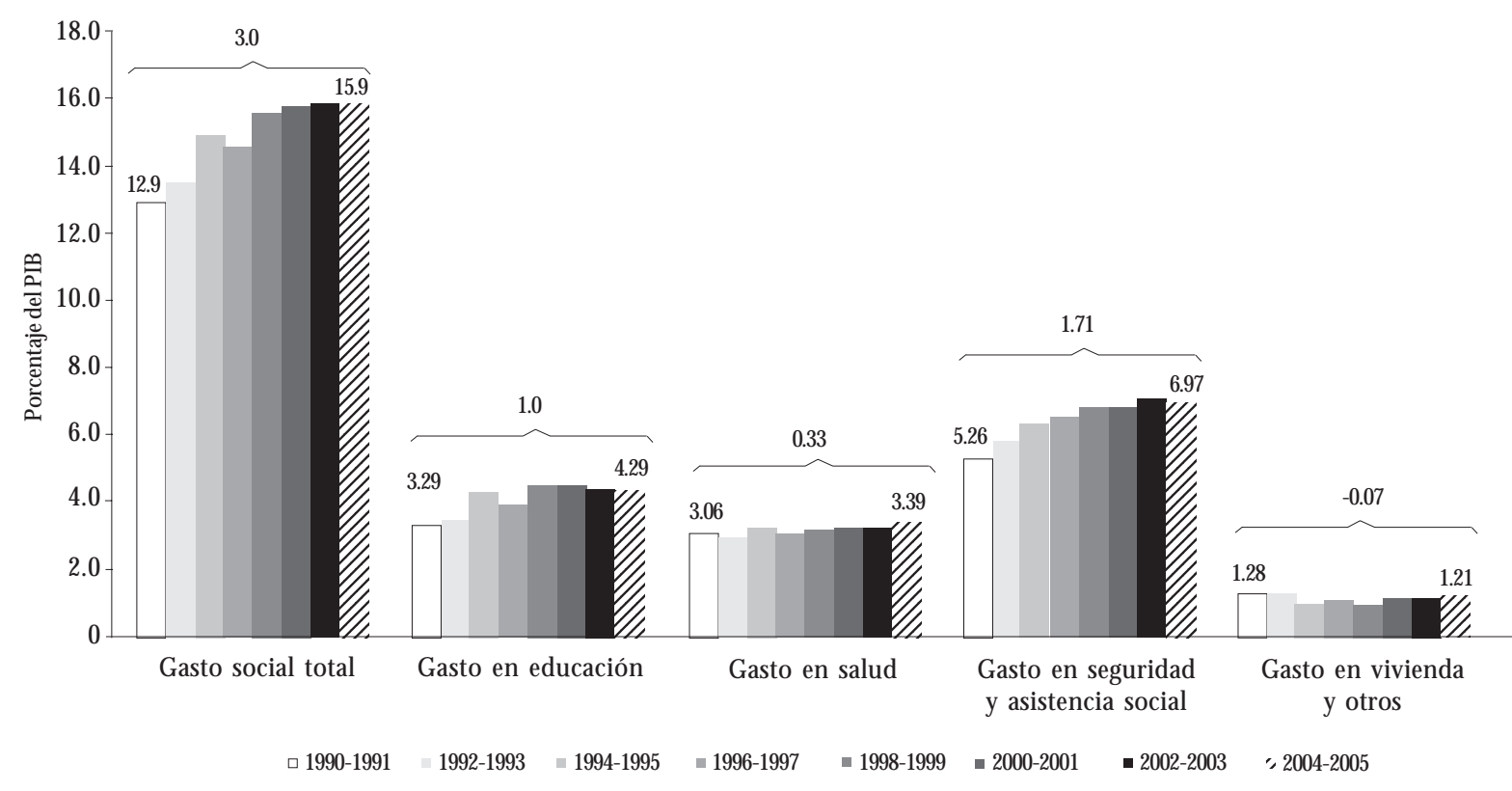

Gráfico 3. América Latina y el Caribe (21 países): evolución del gasto público social como porcentaje del PIB por sectores 1990-1991 a 2004-2005, en porcentajes.

Fuente: CEPAL, con base de datos de gasto social ${ }^{12}$. 


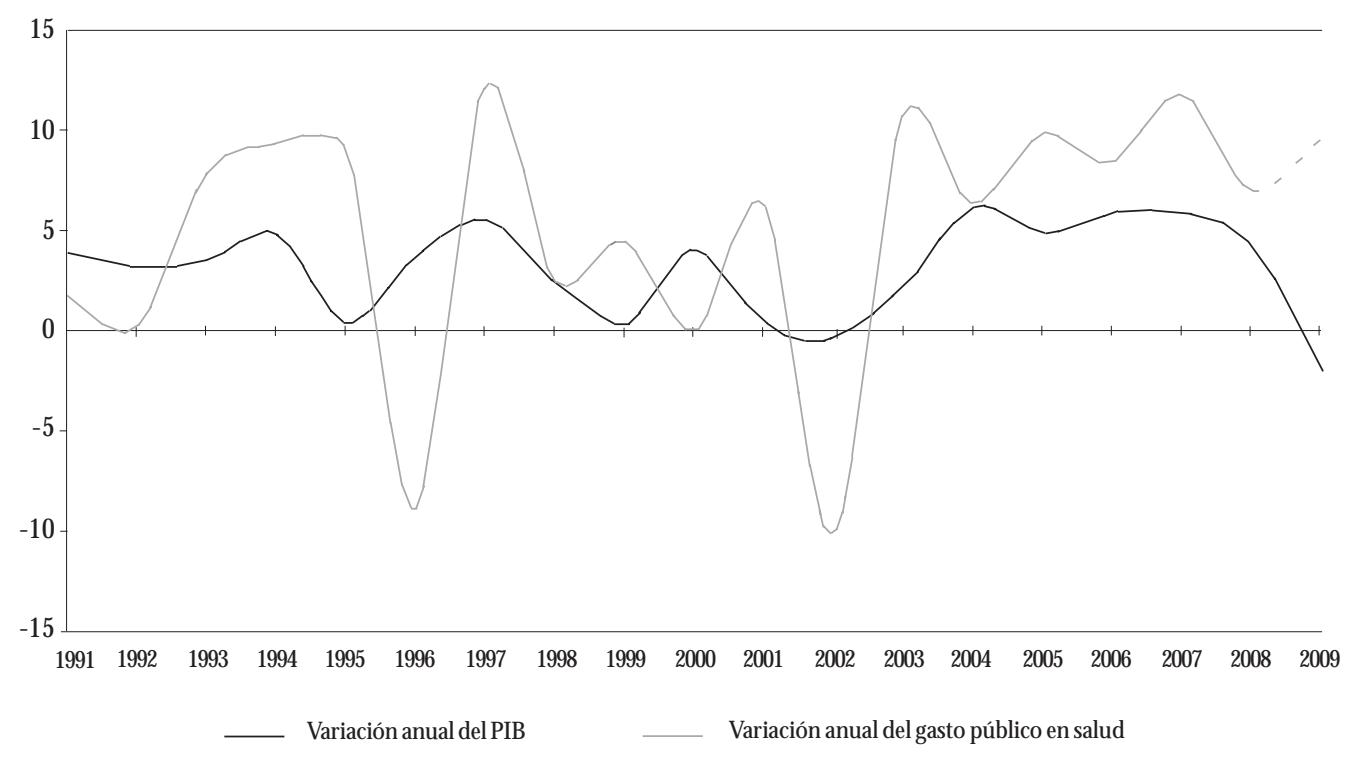

Gráfico 4. América Latina y el Caribe (21 paises): variación anual del gasto público en salud y el producto interno bruto, 1990-2009 (porcentaje de variación).

Fuente: CEPAL, con base de datos de gasto social ${ }^{12}$.

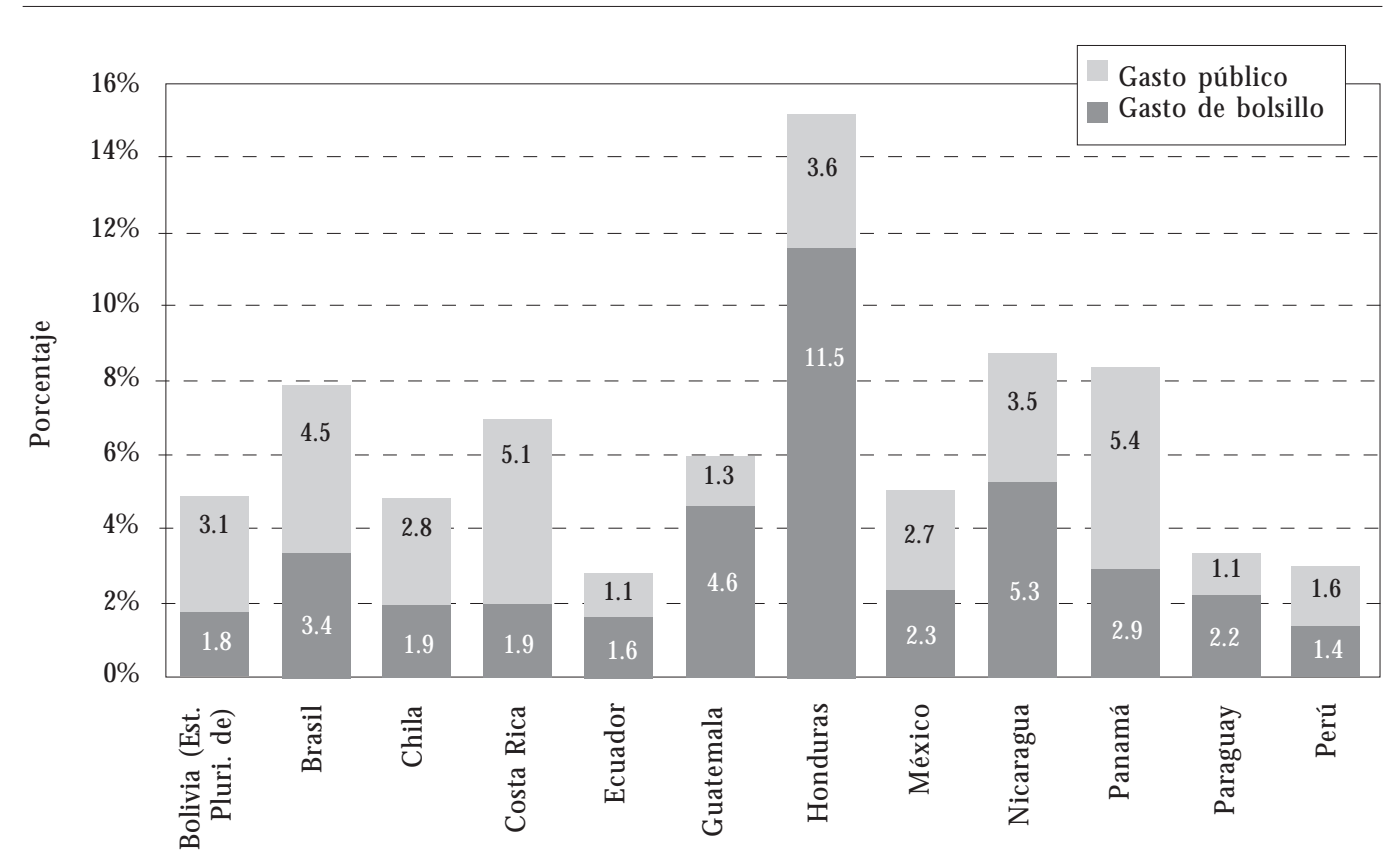

Gráfico 5. América Latina (12 países): gasto de bolsillo en salud y gasto público en salud a/como porcentaje del PIB, 2003/2006.

Nota: a/ Las coberturas del gasto público consideradas son: Bolivia (Estado Plurinacional de), Brasil, Costa Rica, Panamá y Perú: Sector público no financiero o sector público total; Chile, Guatemala y H onduras: Gobierno central; Ecuador, M éxico, Nicaragua y Paraguay: Gobierno central presupuestario.

Nota: El cálculo del gasto de los hogares forma parte de una investigación en marcha sobre gasto de bolsillo en salud realizada por M arcela Perticara y Ana Sojo.

Fuente: Comisión Económica para América Latina y el Caribe(CEPAL), con tabulaciones especiales delas encuestas deingresos y gastos de los respectivos países y de la base de datos sobre gasto social y cifras oficiales de los países ${ }^{12}$. 
Pero la exigibilidad de los derechos sociales, económicos y culturales (DESC) es incremental en el tiempo y requiere perfeccionar instituciones, ampliar la provisión de recursos y avanzar en la redistribución e inclusión sociales. Al ser escasos losrecursos, cabejerarquizar su satisfacción, pero deben incrementarse gradual mente los mínimos garantizables demanera universal, en sintonía con la progresividad de losDESC. Lo ideal es que esos mínimossefijen democráticamentemedianteproceso informado de concertación política, a fin de reducir las tensiones entre lo judicial y lo político en materia de exigibilidad?.

Encarnados en un cuerpo jurídico consistente y operativo, los derechos fijan estándares imperativos para el conjunto de la sociedad y, correlativamente, situaciones inadmisibles. Losinstrumentos jurídicos pueden contribuir a que la sociedad asuma como propios los valores de la solidaridad y reciprocidad; pero no debe sobredimensionarse la judicialidad de los derechos sociales. Aún cuando ventileintereses colectivos, el proceso judicial por su naturaleza tiendea soslayar la multiplicidad de factores vinculados con el diseño de una política social. Las atribuciones centrales en materia del cumplimiento de derechosy la formulación de políticas sociales degran escal a y largo alcance temporal son resorte de los poderes políticos, papel que la judiciabilidad no debe suplantar ${ }^{9,13,14}$.

En el contexto de la discusión sobre cohesión social, la CEPAL ha realizado una importantere flexión acerca de las garantías relacionadas con los derechos sociales, y de las garantías políticas asociadas. De las garantías políticas, deberealzarse la de igualdad y la prohibición de discriminación, los principios de generalidad y universalidad de la ley. En materiadederechos sociales, como complemento dela necesaria progresividad delos avances, la prohibición de regresividad o retroceso que limita la posibilidad de que el Estado reduzca niveles de protección social ya asegurados normativamente. En este marco, puede, además, hacerse efectivo en la región el espíritu universal que contienen principios como los de Limburgo y $M$ aastrich que, si bien admiten que debeconsiderarsela limitación de recursos para eval uar el cumplimiento de las garantías de contenidos mínimos, por otra parte señalan que un Estado puede aducir el no cumplimiento de las obligaciones mínimas por la falta derecursos disponibles, siempre y cuando demuestre haber realizado todos los effuerzos a su alcance para utilizar todos los recursos disponibles en pos de satisfacer, con carácter prioritario, tales obligaciones ${ }^{9,14}$.
Prestaciones de salud y universalidad: Colombia, Chiley M exico

Colombia: complejidad del sistema y judicialización del derecho a la salud

La Constitución de 1991 y la Ley 100 de 1993 buscaron lograr la cobertura universal unificando el régimen de beneficios y obligaciones en un marco de descentralización del gasto, queimplantó una competencia regulada entre aseguradores y prestadores en un sistema de seguridad social integral y solidario. La reforma acontece en un momento de apertura económica y la adopción de reformas que buscaban eliminar los monopolios a cargo del Estado, en el caso de la salud, de aseguramiento y de prestación. Por ello, el establecimiento de un plan explícito de beneficios resulta en parte de una nueva concepción de la organización del sector ${ }^{15}$.

Se distinguió un régimen contributivo -que se nutre de las contribuciones obrero-patronales, que ascienden al $12 \%$ del salario del afiliadodel subsidiado para los más pobres, ambos con prestaciones reguladas por un Plan obligatorio desalud (POS). El POS seconcibió como un plan único, de carácter obligatorio eintegral que, para los afiliados principales, era como mínimo el mismo que tradicionalmente se había asignado al ISS e incluía todos esos servicios de salud, excepto la cirugía estética y la ortodoncia. Se estableció el acceso de los beneficiarios del régimen subsidiado a un plan progresivamente integral que, hasta 2001 debía mejorar de forma escalonada. Se contemplaba a tal fin un aumento progresivo de las transferencias a los municipios destinados a salud, al igual que de los recursos asignados a subsidios de salud.

El sistema de protección del régimen contributivo se unificó en torno al Fondo de Solidaridad y Garantía (FOSyGA), buscando equilibrar recursos y condiciones de equidad mediante una unidad de pago por capitación, ajustada a grupos de edad. Se instauró la cobertura del grupo familiar y se extendió la obligatoriedad de la afiliación hacia todos los trabajadoresindependientes con capacidad contributiva.

La separación de las funciones de financiamiento y provisión fue otro aspecto fundamental. En esta arquitectura, las entidades promotoras de salud (EPS) concentran la función financiera y aseguradora, vertebran el sistema de prestaciones, y cumplen un papel integrador. Son entidades públicas y privadas que, por delegación del Fondo, son responsables del registro de 
los afiliados, del recaudo de sus cotizaciones y queorganizan y garantizan la prestación del POS, debiendo ofrecer opciones de prestadores. Las EPS están obligadas a contraer un reaseguro para cubrir riesgos catastróficosquetienevigencia tanto para el régimen contributivo como para el subsidiado. El plan es brindado por las diversas instituciones prestadoras públicas y privadas de servicios de salud (IPS).

Pero el incremento delas prestaciones del subsidiado ha ocurrido de una manera algo fortuita, integrando al gunas prestaciones de todos los niveles e incluso de las más complejas, debido a la vigencia del reaseguro y a la interposición de tutelas. También las prestaciones del POS se han ampliado en el marco de la judicialización del derecho a la salud. Actualmente el régimen contributivo cubre a un $38 \%$ de la población. Pero solo cotiza el $14 \%$, el subsidiado cubre a $50 \%$ de la población más pobre y en zonas rurales y los no afiliados, que se atienden en hospitales. Los que realizan gastos de bolsillo diferenciales son menos del $12 \%$ de la población. A lo largo del tiempo, el sistema ha experimentado varias tensiones, entre ellas: el financiamiento recae en un tercio de la población que paga sus contribuciones y que también paga impuestos generales; la intermitencia laboral crea un tránsito en doble vía del régimen contributivo al subsidiado; y las acciones detutela generan expectativas deun plan de salud ilimitado ${ }^{16}$.

$\mathrm{H}$ an surgido conflictos sobre la interpretación del alcance del plan de beneficios entre los diferentes actores y se han generado un número creciente de tutelas interpuestas para acceder a los servicios de la llamada "zona gris" negados por lasEPS. Lasinclusiones, afirman análisis, han sido puntuales y sin aparente lógica u orden sistemático, poco transparentey sin orientación clara. En esecontexto fuequela sentencia T760 dela Corte Constitucional (entidad judicial máxima encargada de velar por la protección de los derechos contenidos en la Constitución de 1991) ordenara al M inisterio de la Protección Social precisar el contenido del POS y aclarar dudas que han surgido acerca de su alcance real ${ }^{15}$.

La Ley 100 se adopta pocos años después de que la Constitución de 1991 redefiniera el papel del Estado, estableciendo que éste no solo debía reconocer los derechos de los ciudadanos, sino quedebía garantizarlos. El mecanismo de la tutela fue introducido por la Constitución para fortalecer el cumplimiento del derecho a la salud medianteun procedimiento ágil y rápido, cuando estos resulten amenazados. H asta agosto de 2008 la seguridad social no se consideraba como un derecho fundamental, y sele protegía por conexidad con otros, por tratarse desujetos con especial protección constitucional y dondese trateel derecho a la salud como subjetivo. En aquel marco, cuando a una persona se le niega un servicio incluido en el POS, puede interponer una "tutela"; de igual forma para solicitar servicios excluidos del POS cuando la negación amenace alguno de los derechos fundamentales. Si el juez falla a favor del ciudadano, la EPS debeprestar la atención con cargo a los recursos del Estado y en particular al FOSyGA ${ }^{16}$. Crecientemente, ha sido permitido solicitar servicios no comprendidos en el POS, lo cual no sólo ha desdibujado sus límites, creando un POS "judicial" o "paralelo", sino que ha puesto en jaquela sustentabilidad financiera del sistema ${ }^{15}$.

U primy ha distinguido períodos en el desarrollo de la jurisprudencia constitucional. Antes de 1992, poco eficaz, cuando la salud se considera un derecho programático; entre 1993 y 1994 se establece por conexidad el derecho a la salud fundamental; 1994-2003, seexpandelanoción deconexidad, con lo cual se genera un activismo de la tutela individual; desde 2003 se avanza hacia la consideración como derecho fundamental y aunque no se afirma que todo sea posible de reclamar, se incrementan las demandas por servicios no POS ${ }^{16}$. Entre 2003 y 2005, las tutelas crecieron un $68 \%$. A fines de 2009 , cerca del $60 \%$ de ellas corresponden a salud y pensiones y de ese conjunto, $75 \%$ a salud. El monto de las tutelas por servicios, procedimientos o medicamentos no incluidos en el POS, alcanzaron un valor aproximado al $0,25 \%$ del PIB y fueron recobradas al FOSyGA. En el 2007 se realizaron 1 millón de recobros ( 120 millones deserviciosal año). Desdeel 2002 , con un gran crecimiento a partir del 2003, la tutel a discrimina a favor del régimen contributivo - del total sieteveces más correspondían a este. Además, se conceden crecientemente beneficios adicionales, no comprendidos en el POS ${ }^{16}$.

A parte de las necesidades de salud y la negación de servicios por parte de los aseguradores, algunos autores afirman quela indefinición delas coberturas del Plan O bligatorio de Salud y la falta deactualización integral delas tecnologías usadas en los tratamientos - por lo menos en losúltimos 20 años- explican las tutelas ${ }^{17}$. Entre otros actores, entidades como FEDESARRO LLO han señalado que la falta deactualización periódica del POS conforme a las características epidemiológicas cambiantes dela población unidaal activismo judicial han llevado a una situación en la queel contrato de aseguramiento no es transparenteni exi- 
gible ni para las empresas aseguradoras ni para los usuarios, y arguyen queel crecimiento desmesurado de los pagos no POS indica que el activismo judicial ha desbordado delejos cualquier omisión gubernamental al respecto ${ }^{18}$.

En agosto de 2008, la Corte Constitucional ordenó aclarar, actualizar y unificar los dos planes de beneficios y estipuló que, a partir de este momento, el derecho a la salud esun derecho fundamental autónomo y, por ende, tutelablesin necesidad de demostrar la conexión con algún otro derecho fundamental, siempre y cuando los serviciosque se soliciten estén avalados por el médico tratante.

La magnitud delastensiones definanciamiento Ilevó en enero del 2010 a queel Gobierno expidiera 12 decretos dentro del marco de la Emergencia Social para encarar a corto plazo el desfinanciamiento del sistema y quehan generado gran polé mica. Sus detractores se refieren tanto a su contenido como a su carácter inconsulto, y por no haber utilizado el conducto regular del Congreso ${ }^{17}$.

La declaratoria de emergencia social aduce una mezcla de situaciones sobrevinientes, agravamiento súbito de problemas antiguos y cuestiones estructurales. Por una parte, un aumento extraordinario de solicitudes de medicamentos y servicios no contemplados en el POS que pondría en inminente riesgo de agotarse la cuenta de compensación del régimen contributivo: dado que esa cuenta es la columna vertebral de sistema, deentrar en problemas deinsuficiencia, todo el sistema presentará fallas crecientes. Por otra, un creciente déficit de los Departamentos quecarecen de recursos financieros para responder por gastos no POS del régimen subsidiado, ya quelas transferencias del Sistema general de participaciones (SGP) destinadas al pago delos servicios a cargo de los departamentos han venido cayendo, mientras que sus responsabilidades han ido en aumento. Además un aumento de la cartera de las EPS y las IPS ponía en riesgo su liquidez por corrupción, por un monto inexplicable de recobros por medicamentos, por un lento flujo de recursose insuficiencia en la vigilancia ${ }^{17}$.

$\mathrm{H}$ ay medidas orientadas a arbitrar recursos adicionales y otras a racionalizar el gasto y destrabar recursos para mejorar su liquidez y controlar los gastos no POS. Se aduce que permitir que los gastos continúen aumentando más allá de las capacidades de financiación pondría en peligro la atención básica de la población asegurada ${ }^{17}$.

Por tanto se encaran debilidades institucionales y políticas, ya que parece haberse carecido del contrapeso de una entidad técnica, capaz de emi- tir conceptos independientes sobre la indicación clínica de lo solicitado y la capacidad del sistema de financiarlo. Así, los límites establecidos en un plan explícito han perdido gradualmente su significado, peligrando derivar hacia un financiamiento sin priorización, pero inmerso en un sistema que tiene al tos costos de transacción.

De allí que Colombia esté entrando en una nueva fase de discusión respecto de la priorización, en la cual los planes de beneficios de salud están en el centro de los debates. La explosión de la solicitud de servicios por fuera del POS y su amenaza para la sustentabilidad financiera del sistema y la sentencia de la Corte Constitucional que transforma el derecho de salud en un derecho fundamental obligan a aclarar, actualizar y unificar el plan de beneficios ${ }^{15}$. Todo ello es neurál gico en la reforma que actualmente se discute en el parlamento sobre aspectos relativos a sostenibilidad del sistema, a su universalidad, y a la necesidad de fortalecer la institucionalidad de los planes de beneficiosy del proceso deunificación.

Chile: prestaciones avanzadas

como rearticulación del sistema dual

Posibilitada por el contexto autoritario, la radicalidad de la reforma emprendida en los ochenta no tieneparangón en el mundo en cuanto a la dualidad del sistema de salud entronizada.

El aseguramiento compulsivo es propio de los llamados seguros sociales, públicos o nacionales, que buscan una diferenciación de riesgos estable y solidaria en el seno de combinaciones público privadas. De allí que la lógica dual del sistema de salud chileno sea única en el plano internacional: la cotización obligatoria, a cargo exclusivo del trabajador, le permite afiliarseal sistema público desalud medianteel Fondo Nacional deSalud (FONASA) cuya racionalidad dereparto favorece la solidaridad, o a las instituciones de salud previsional (ISAPRES) que, a pesar del carácter compulsivo del aseguramiento, funcionan bajo una lógica de seguro privado asociada al riesgo individual.

EI FONASA da acceso a prestaciones públicas en la llamada modalidad institucional o a las privadas mediantela modalidad delibreelección, que está sujeta a diversos copagos, y además de las contribuciones de sus afiliados se nutre también de otros aportes con cargo al presupuesto público. Al contrario de la lógica solidaria característica del aseguramiento compulsivo, las cotizaciones obligatoriasquesecanalizan a las ISAPRESseplasman en planes individualesque, al renovarsecada 
año, permiten una selección de riesgo, al ajustarse los precios y la cobertura a la edad, riesgo en salud y sexo del asegurado. Las ISAPRES cubren a cerca del 30\% de la población y la elevación de sus precios ha superado con creces la de otros sectores de la economía. El sector público, desprovisto de barreras deentrada, cubrea la población demenores ingresos y a la de mayores riesgos y cumple una función de reaseguro global del sistema.

Sabida es la dificultad política inherente a la exclusión explícita de prestaciones por parte del asegurador público en salud. Chile resulta también bastante singular en este ámbito, ya que el FONASA cuenta con un catálogo explícito de prestaciones, disposición quesiendo polémica por naturaleza, también se vio facilitada por el contexto autoritario dela reforma del gobierno militar. Pero cabe destacar que desde la década del noventa el catálogo de prestaciones ha aumentado sustancialmente, incluyendo las dealto costo queseconsolidan con las prestaciones garantizadas.

La garantía de prestaciones avanzadas de salud (GES) que se pone en efecto en el año 2005 (más conocidas como Plan Auge), tras un complejo proceso denegociaciones políticas ${ }^{19}$, modifica parcialmente la morfología dual del sistema y comprende fundamentalmente las avanzadas y de alto costo. Aunque no altere la solidaridad del financiamiento a escala del sistema y las dos lógicas del aseguramiento, imprime solidaridad al sistema de salud y es un avance contra la dualidad al asegurar el acceso efectivo, uniforme y obligatorio a algunas prestaciones, tanto para el sistema público como para el privado. Para el asegurado del sistema público, se incrementa y garantiza la cobertura efectiva de al gunas prestaciones, incrementando la certeza en cuanto al logro de las prestaciones que están sujetas además a cumplirseen condiciones de oportunidad y calidad. En el caso de las ISAPRES, éstas fijan precios de acuerdo con el tipo de prestadores elegidos, lo cual evidentemente estratifica las prestaciones según la capacidad de pago y que se asocian con diferencias de calidad y de hotelería.

Los beneficios son irrenunciables, es decir, ningún afiliado puede oponerse a que les sean incorporados a sus contratos de salud. Las garantías encarnan un plan de salud único, con las mismas coberturas, con una reglamentación de copagos y de las obligaciones. Consideran el acceso, la calidad, la protección financiera y la oportunidad: el acceso, ya que es obligación de FONASA y las ISAPRES otorgarlas; calidad, ya que deben otorgarse por prestador registrado 0 acreditado; oportunidad, ya que se fija plazo máxi- mo para disfrutarlas; protección financiera, ya que se regulan los copagos y la gratuidad. Vista en el concierto latinoamericano, es esta una de las experiencias más avanzadas, ya que las garantías tienen una especificación mucho mayor sobre los alcances, obligaciones, sujetos deudores de estos derechos y los instrumentos de garantía o de protección.

Se incluyen algunas patologías cuya cobertura en la atención pública había sido muy baja, tales como infarto del miocardio, cáncer de mama, cáncer gástrico, cuidado paliativo del cáncer terminal, VIH/SIDA, neumonías del adulto mayor ehipertensión arterial. Tanto las ISAPRES como FONASA deben brindar coberturas estipuladas a sus afiliados, proceso que es supervisado por la Superintendencia de Salud. Además, ningún plan de las ISAPRES podrá, en adelante, otorgar una cobertura inferior a las prestaciones consideradas en la modalidad de libreelección de FONASA. LasISAPRES no pueden denegar estas coberturas aduciendo preexistencias, aunque puedan determinar una red de prestadores y las condiciones en que ésta brindará las atenciones. Ello es fundamental como superación parcial de la selección de riesgo que tradicionalmente ejercían al suscribirse los planes anuales.

Se fijan reglas de responsabilidad del Estado en materia sanitaria, debiendo responder los órganos de la administración por "falta de servicio". H asta ahora no se advierte en Chile una judicialización del derecho a la salud, ya que la canalización de los reclamos está contenida dentro de cauces administrativos.

Los indigentes y carentes de recursos beneficiarios de FON ASA gozan degratuidad en las atenciones que reciban. Todos los demás asegurados, tanto de ISAPRES como de FONASA, contribuyen hasta con un $20 \%$ en el pago de las prestaciones de acuerdo al arancel que establezca las garantías explícitas. Sefijan por ley una amplia gama de copagos progresivos respecto de los ingresos y que consideran la severidad del gasto en que se incurre según el tipo y gama de enfermedades, teniendo en consideración al grupo familiar.

Para tener derecho a las garantías explícitas, los beneficiarios dela atención pública deben acceder a la red asistencial en el nivel de la atención primaria, salvo casos de urgencia o emergencia calificados. Pero los ben eficiarios podrán acceder al nivel de atención correspondiente cuando el diagnóstico se efectúeen un prestador fuera dela red pública y en convenio con el FONASA. Los beneficiarios de la atención privada deben atenderse en la red convenida con la ISAPRE. 
La reforma, además, repercute en la gestión, al establecer protocolos para cada uno de los tratamientos, lo queevita la dispersión y afianza que sebasen en evidencia. Los desafíos son, entreotros, mejorar la gestión, tener sistemas de alertas y detección de carencias, afiatar las redes de prestación y la entrega de recursos a los prestadores, establecer ajustes en las prestaciones (por ejemplo, las atenciones en prematurez vinculadas al AUGE fueron mayores que las proyectadas).

El aseguramiento privado encara significativas caí das dela rentabilidad, los afiliados tienden a estancarse eincluso a caer ya quela selección de riesgo ha propiciado un éxodo al sistema público. El sistema privado sufrió un fuerte cuestionamiento por parte del Tribunal Constitucional, que declaró inconstitucional quelas tablas defactores que determinan el costo delos planes delos diversos grupos de beneficiarios sean definidas mediante normas administrativas. H ay esfuerzos por reestructurar los modelos de negocio de la salud privada e introducir cambios en la integración vertical entre las ISAPRES y los prestadores privados. En ese contexto, la compra de servicios por parte del sector público puede dar nuevos aires al sistema privado y está en la agenda del nuevo gobierno ${ }^{20}$.

La salud pública enfrenta también situaciones críticas. Pese al fuerte aumento del gasto, hay un grave déficit operacional, se requieren nuevos hospitales, hay un serio déficit de especialistas, las listas de espera superan los 300.000 pacientes y el éxodo de afiliados con mayores riesgos desde las ISAPRES incrementa los problemas. Se arrastran graves problemas de gestión, de viejas prácticas como el uso parcial dela capacidad instalada y dificultades en la gestión del personal ${ }^{20}$.

Las orientaciones básicas de la política de salud del Gobierno de Piñera contemplan: reducir las listas de espera, enfatizan do la compra de servicios al sector privado, lo que puede incrementar significativamente los déficits financieros del sector público; otorgar bonos que permitan la elección de prestadores públicos o privados; promover la competencia entre las instituciones de salud; transformar los hospitales y consultorios en sociedades anónimas hospital arias abiertas a la asociación con entes privados y constituidas con participación minoritaria del Estado, dando opción preferente a sus funcionarios; fortalecer y acelerar la concesión al sector privado para la construcción y operación no clínica de los hospitales públicos ${ }^{20}$.
México: seguro popular

y ampliación de cobertura

Con el Seguro Popular de Salud (SPS) seamplía en M éxico la cobertura del aseguramiento que incluyegarantías de financiamiento, pero no modificala segmentación: adiciona recursos propios, canal izados de manera muy compleja y abarca una gama de intervenciones que es menor a la cobertura global de riesgos de la seguridad social. Involucra modificaciones institucional es que implican fortalecer centros públicos de salud, modifica la combinación público-privada porque abre más participación a los prestadores privados y tiene como desafío fortalecer la capacidad de regulación de calidad.

Las garantías de financiamiento del SPS están respaldadas por recursos propios adicionales, canalizados a los prestadores y que provienen fundamentalmente del presupuesto federal y de las entidades federativas. La complejidad del financiamiento pone sobre la mesa un asunto no trivial, que constituye un reto en términos institucionalesy organizativos: a saber, cómo los prestadores logran usar y combinar eficientemente diversos recursos para los que rigen singulares mecanismos de acceso, criterios de asignación, regímenes presupuestarios, tipo de transferencias, programación y ejecución de los flujos de recursos, e incentivos implícitos o explícitos en términos de la gestión que deben conciliarse ${ }^{19}$.

El SPS naceen el año 2002 como plan piloto y se inicia oficialmente en el 2003 a cargo de la Subsecretaría de Prevención y Promoción de la Secretaría de Salud, que reúnela función decompra. Se implanta para ampliar la cobertura de aseguramiento público, reducir el pago de bolsillo y fomentar una atención oportuna. De carácter voluntario y sin barreras de acceso, tiene como objetivo la población denominada abierta, es decir, la que no cuenta con ningún tipo de seguridad social. Las aportaciones de las familias son progresivas respecto del ingreso.

El seguro tieneportabilidad y comprendeuna cobertura estandarizada, mediante un paquete explícito deintervenciones costo-efectivas, incluidos medicamentos. Cubre al asegurado directo, al cónyuge, los hijos hasta los 18 años de edad y a los padres mayores de 65 años de edad que sean dependientes económicos. La cobertura comprende vacunas, consultas de medicina en general, exámenes físicos de hombres y mujeres, diagnóstico y tratamiento de 39 enfermedades, atención dental, métodos de planificación familiar, atención en salud reproductiva en las distin- 
tas etapas de la vida, diagnóstico y tratamiento defracturas, y 16 procesos quirúrgicos de distinta naturaleza. El paquete supera los criterios minimalistas de extensión de cobertura característica de programas previos, que incluye atenciones de segundo nivel y atención dealgunas enfermedades catastróficas ${ }^{21}$.

Para evitar y acotar interpretaciones divergentes sobresu al cance, el catál ogo CAUSES abarca un listado detallado de intervenciones concretas y especifica los servicios y tratamientos asociados. El paquete del Fondo de servicios catastróficos (FPGC) abarca un catálogo de enferme dades quecuentan con protocolo deatención clínica, usado para estimar su costo promedio y describe los medicamentos necesarios, las prue bas de laboratorio y gabinete. La Comisión $\mathrm{Na-}$ cional de Protección Social en Salud (CNPSS), órgano rector del SP a nivel federal, es responsable de la definición del CAUSES. En el caso del FPGC, la definición del plan debeneficios es complementada por la intervención del Consejo de Salubridad General (CSG), entidad interinstitucional que tradicionalmente ha estado a cargo del desarrollo de protocolos de atención en enfermedades de alto costos, en la que participan las principales instituciones del sector ${ }^{15}$.

EI SPS tieneimplicaciones respecto dela combinación público privada del sistema de salud mexicano. De acuerdo con la normativa, los estados pueden adquirir servicios del sector priva- do. Con la ampliación de cobertura provista por el nuevo aseguramiento, se posibilita una mayor participación del sector privado en la prestación deservicios. Con el SPS, el IM SS también se convierte, según las circunstancias, en un posible proveedor de servicios. La atención se brinda en los centros de salud del sistema de la Secretaría de Salud que estén en capacidad de proporcionar servicios con determinados estándaresy quecuenten con recursos humanos e infraestructura, lo cual se evalua mediante una acreditación obligatoria de los prestadores de servicios. Además, las instituciones que provean atención bajo el Fondo de servicios catastróficos (FPGC) que cubre patologías de al to costo, también necesitarán estar certificadas ${ }^{19}$.

La coexistencia de diversos sistemas de aseguramiento va de la mano de la competencia por los recursos. En el caso del SPS, su creación ha redundado en la disminución de la partida de recursos públicos destinados a los derechohabientes de la Seguridad Social ${ }^{15}$. Se ha señalado un aspecto de la reforma que evidencia incentivos contradictorios respecto de la combinación público-privada, debido a que muchos médicos del sector público tienen también consultas privadas. Deallí quela regulación debiera velar porque este elemento no conduzca a conductas de riesgo moral en detrimento dela amplitud y calidad de las prestaciones públicas para ampliar la participación privada22. 


\section{Referencias}

1. Whitehead M. Conceptos y principios de igualdad y salud. Madrid: M inisterio de Sanidad y Consumo; 1990.

2. Sojo A. La trayectoria del vínculo entre políticas selectivas contra la pobreza y políticas sectoriales. Rev CEPAL 2007; 91:111-131.

3. Gutiérrez JP, Leroy JL, DeM aria L, Walker D, Campuzano JC, M oreno K, Serván E, Sosa Rubí SG, Bertozzi SM. Evaluación de la calidad de los servicios de atención de salud asignados a la población beneficiaria de oportunidades. In: Comisión Económica para América Latina (CEPAL). Seminario Internacional: Desigualdad Socioeconómica y el Derecho a la Salud en América Latina y el Caribe en una Perspectiva Internacional; 2008; 3 y 4 de nov.; Santiago de Chile, 2008. Santiago de Chile: CEPAL; 2008. [acessado 2011 abr. 28]. Disponible en: http://www.eclac.cl/id.asp?id=34175

4. Organización Panamericana de la Salud (OPAS). Ampliación de la protección social en materia de salud: iniciativa conjunta de la Organización Panamericana de la Salud y la Organización Internacional del Trabajo (CSP26.R19). In: XXVI Conferencia Sanitaria Panamericana; Washington, D.C., 23 al 27 sep., 2002. Washington, D.C.: OPS; 2002.

5. Chile. Ministerio de Salud de Chile. Evaluación del estado de avance en el cumplimiento de objetivos sanitarios y de metas destinadas a disminuir las desigualdades en salud. Santiago de Chile: Ministerio de Salud de Chile; 2006.

6. Flórez CE, Soto VE, Acosta OL, Kart C, M isas JD, Forero N, Lopera C. Avances y desafíos de la equidad en el sistema de salud colombiano. Bogotá (CO): Fundación Corona; 2007. (Documentos de trabajo, 15).

7. Carrera F, Castro M, Sojo A. Progresar hacia la equidad: sinopsis de algunos retos del financiamiento de la salud y las pensiones en Guatemala, Honduras y Nicaragua. In: Prado A, Sojo A, editores. Comisión Económica para América Latina (CEPAL). Envejecimiento en América Latina: sistemas de pensiones y protección social integral. Santiago (CL): CEPAL; 2010. p. 41-64. (Libros de la CEPAL, n. 110).

8. Comisión Económica para América Latina (CEPAL). La protección social de cara al futuro: acceso, financiamiento y solidaridad. Santiago: CEPAL; 2006. (M ontevideo, Sesión 31: 20-24 marzo de 2006).

9. Comisión Económica para América Latina (CEPAL). La cohesión social: inclusión y sentido de pertenencia en América Latina y el Caribe. Santiago: CEPAL; 2007.

10. Comisión Económica para América Latina (CEPAL). Objetivos de desarrollo del milenio: la progresión hacia el derecho a la salud en América Latina y el Caribe. Santiago: CEPAL; 2008. [acessado 2001 abr 28]. Disponible en: http://www.eclac.org/publicaciones/xml/ 4/33064/2008-808-ODM SaludRev-1indd.pdf

11. Barcellona P. A proposito della cittadinanza sociale. Democrazia Diritto 1988; (anno XXVIII):2-3.

12. Comisión Económica para América Latina (CEPAL). Panorama social de América Latina, 2010: documento informativo. Santiago: CEPAL; 2011.
13. Courtis C. Los derechos sociales en perspectiva: la cara jurídica de la política social. In: Sojo A, Uthoff A, compiladores. Desempeño económico y política social en América Latina y el Caribe: Ios retos de la equidad, el desarrollo y la ciudadanía. M éxico: Fontamara; 2006. p. 90.

14. Abramovich V, Courtis C. El umbral de la ciudadanía: el significado de los derechos sociales en el Estado social constitucional. Buenos Aires: Del Puerto; 2006.

15. Giedion U, Panopoulou G, Gómez-Fraga S. Diseño y ajuste de los planes explícitos de beneficios: el caso de Colombia y M éxico. Santiago: CEPAL; 2009. (Serie Financiamiento del desarrollo, n. 219).

16. Acosta OL. La judicialización del derecho a la salud en Colombia. In: Comisión Económica para América Latina (CEPAL); Instituto Centroamericano de Estudios Fiscales (ICEFI); Analytical Spectral Devices, Inc. (ASDI); Agencia Española de Cooperación Internacional para el Desarrollo (AECID), editores. Panel IV: Perspectivas de los Sistemas de Salud en Seminario Internacional Repensar lo Social en Tiempos de Crisis; 2009 may 28-29; Antigua, Guatemala: CEPAL; 2009 [acessado 2011 jan]; [34 Diap]. Disponible en: http://www.eclac.cl/dds/noticias/paginas/2/36122/OlgaLuciaAcosta.pdf

17. Ramírez Moreno J. La emergencia social en salud: de las contingencias regulatorias a la desilusión ciudadana. Rev Gerenc Políticas Salud 2010; 9(Supl.18): 124-143.

18. Saavedra V, Sabogal A. La emergencia social: conclusiones de un debate. Economía y política: análisis de la coyuntura legislativa 2010; fev [acessado 2011 abr 29]; (59). Disponible en: http://www. politicaspublicasysalud.org/documentos/sistemade-salud-en-colombia/vi ewdownload/16-sistemade-salud-en-colombia/34-la-emergencia-socialconclusiones-de-un-debate.html

19. Sojo A. Health benefits guarantees in Latin America: equity and quasi-market restructuring at the beginning of the millennium. M éxico: ECLAC; 2006. (Serie Estudios y perspectivas, n. 51).

20. Rivera Urrutia E. Política y equidad en el bicentenario: el caso de la política social bajo la Administración de Sebastián Piñera. Santiago: In: Rivera Urrutia E. Santiago: BPE una nueva forma de gobernar: la instalación. Santiago: Fundación Equitas y Fundación Friedrich Ebert; 2010. p. 60-79.

21. Nigenda G. El seguro popular en M éxico: desarrollo y retos para el futuro. Washington, D.C.: Banco Interamericano de Desarrollo (BID); 2005. (N ota Técnica de Salud, n. 2/2005).

22. Organisation for Economic Co-operation and Development (OECD). Estudios de la OECD sobre los sistemas de salud: M éxico. Ciudad de M éxico: OECD Publishing; 2005.

Artigo apresentado em 05/01/2011

A provado em 28/02/2011

Versão final apresentada em 05/03/2011 